\title{
Concealed In the Cavity, Revealed upon Surgery- A Rare but Critical Entity “Gossypiboma”
}

\author{
Maryum Firdaus* \\ Maryum Firdaus, Combined Military Hospital, Pakistan \\ *Corresponding author: Maryum Firdaus, Combined Military Hospital, Rawalpindi, Pakistan
}

\section{ARTICLE INFO}

Received: 幽 March 19, 2020

Published: 慧 April 02, 2020

Citation: Maryum Firdaus. Concealed In the Cavity, Revealed upon Surgery- A Rare but Critical Entity "Gossypiboma". Biomed J Sci \& Tech Res 26(5)-2020. BJSTR. MS.ID.004417.

\begin{abstract}
A female patient 32yrs old G3P002 presented to the hospital OPD with the complaints of pain in the lower abdomen after a cesarean section three days back from a healthcare facility at 37 weeks and 4 days due to fetal distress and cord around the neck. She had attended 03 antenatal visits at the same health center wherein the fundal height was documented at each visit and routine care given. Except for occasional painful fetal kicks, she was otherwise well throughout the antenatal period. On admission to our unit, she had stable vital parameters. Abdomen was markedly tender in the left iliac fossa. Wound examination was unremarkable. Baseline investigations were advised along with an abdominal ultrasound examination. Laboratory investigations were normal except TLC count of $160000 / \mathrm{mm}^{3}$ with neutrophils $90 \%$. Ultrasound showed an echogenic linear structure with dense posterior shadowing in left iliac fossa (as shown in Figures 1-3) with moderate fluid in Pouch of Douglas. A suspicion of some foreign body was made and X-ray abdomen was advised.
\end{abstract}

\section{Introduction}

Gossypiboma is a term with origin from a Latin word "Gossypium" meaning "cotton" and Sawahii word "Boma" meaning "Place of concealment" while another opinion about derivation is that it is derived from Latin word "Gossypium" meaning "Cotton" and a suffix "-Oma" meaning "A tumor or a growth" because most of the cases present as a mass containing cotton matrix within it [13]. It is also known as "Textiloma" or "Cottonoid" is the term used to describe the presence of a cotton matrix retained in the body cavity during surgery. It is proposed to have a worldwide incidence ranging between $0.1 \%$ to $0.3 \%$ of all surgical interventions [4] Most of which are related to abdominal surgeries having an incidence between $0.06 \%$ to $0.1 \%$ [4]. Major risk factors associated with gossypiboma include: emergency operations, Increased BMI of the patient, Unexpected change in operative procedure, omission of the standard sponge count before and after surgery [5]. Other risk factors include multiple teams performing operations on the same patient or patient undergoing more than one operation at the same time [5]. Few risks are also associated with prolonged surgery time, Fatigue inexperience of the staff, Prolonged bleeding during the surgical procedure [5]. Pathologically two types of foreign body reactions have been documented so far in these cases which include an exudative reaction that presents earlier with symptoms like an abscess, fistulous communications, etc and an aseptic fibrinous reaction with delayed variable presentations in the form of adhesions, masses granulomas or pseudotumors[6].

Although the presentation is variable depending upon the following few factors:

a) The time elapsed between surgery and the appearance of symptoms.

b) Location of the foreign body

c) Type of reaction

In case of acute presentation symptoms can be tenesmus, diarrhea or ileus due to irritation of the gut, unexplained pain at or near the surgical site, abscess due to infected foreign body or sepsis as a result of the dissemination of infection whereas chronic cases present with solid palpable masses due to granulomatous response, weight loss, anorexia or vomiting due to intestinal obstruction or a malabsorption type syndrome resulting from fistula formation or intraluminal bacterial overgrowth. Few cases may present as visceral or bowel perforation or sepsis leading to 
death if not treated timely $[7,8]$. Rarely these foreign bodies may migrate from the surgical site into the luminal visceral organs like ileum, stomach, duodenum, colon or bladder without any evident opening in their walls $[9,10]$. In the case of intrathoracic textilomas, presentation is with cough chest pain referred to shoulder, cough, hemoptysis or bilious expectoration. Textilomas left in spinal surgeries may present as low backache, edema, periosteal reaction and neuropathic pain [11].

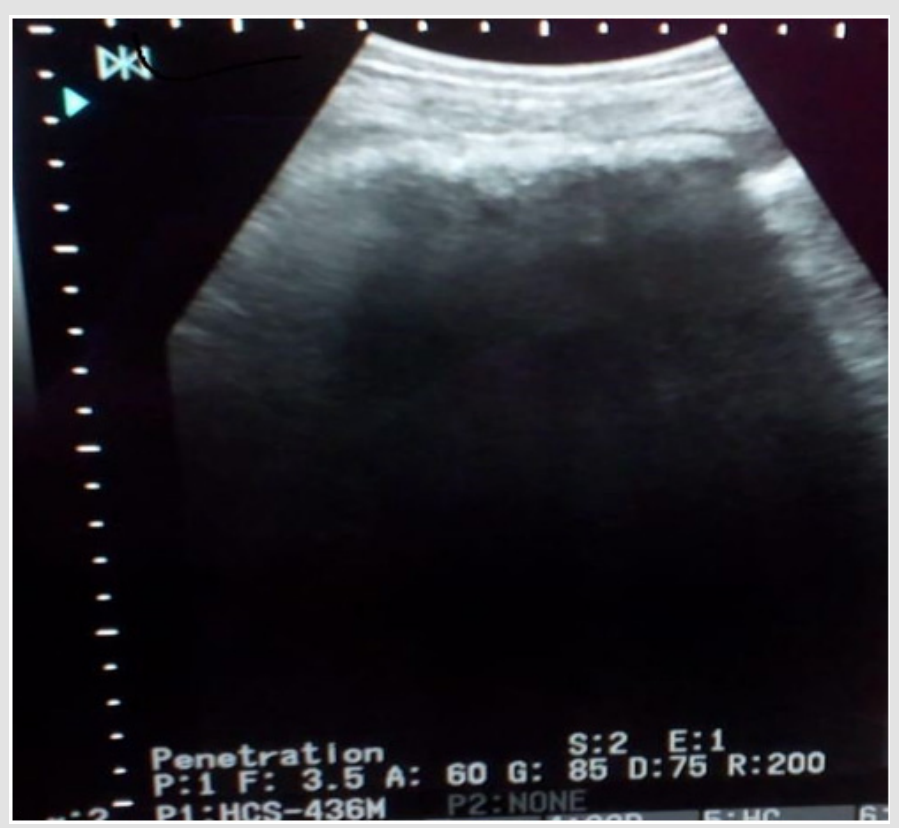

Figure 1: X-ray was done on the same day and showed a dense radiopaque shadow anterior to the left iliac bone representing a metal foreign body. Considering the recent history of the Cesarean section a diagnosis of retained foreign body was made.

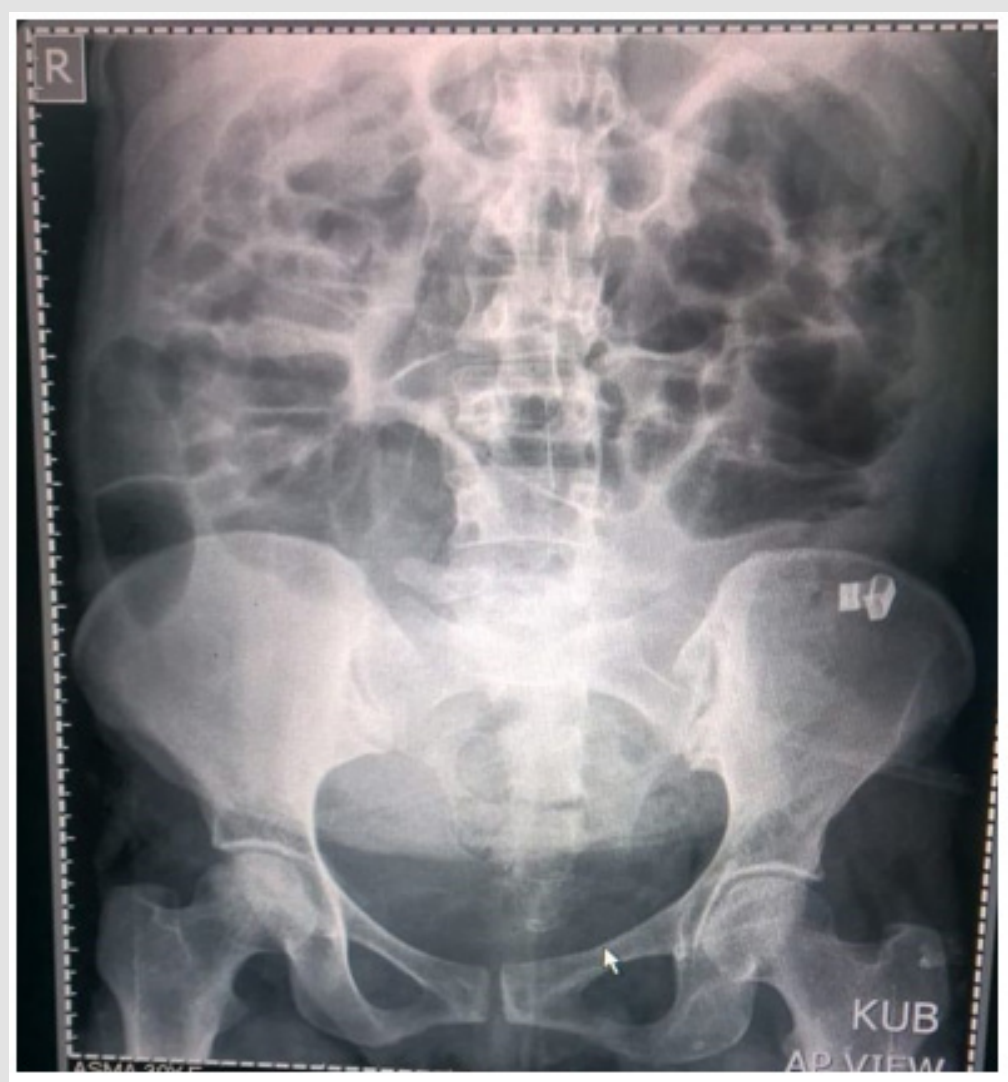

Figure 2: Further CT scan pelvis was advised prior to surgery which showed a well-defined low density mass adjacent to uterus in left iliac region showing internal air loculi and a metallic marker suggestive of gossypiboma. 

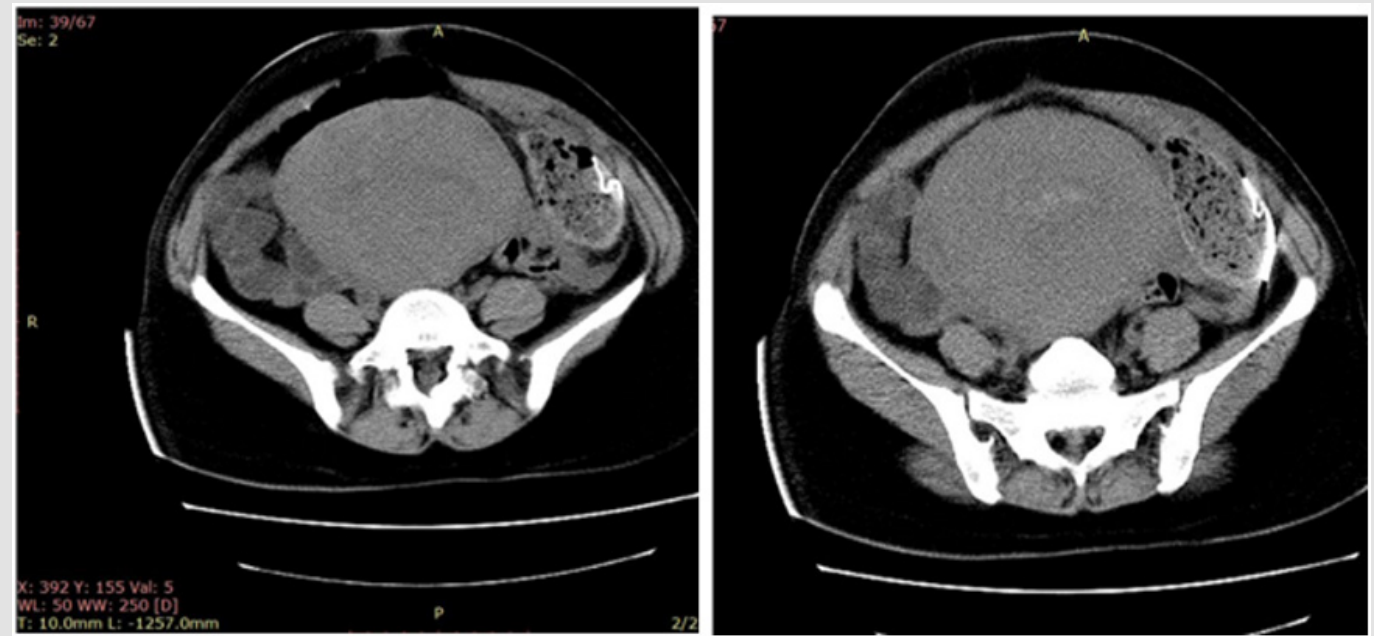

Figure 3: Open laparotomy of the patient was done and the retained abdominal sponge was removed thus confirming the diagnosis of gossypiboma.

Symptomatic cases usually are diagnosed early when radiological investigations are carried out while asymptomatic patients may present decades later or even incidentally. Both radiologic and pathologic diagnostic investigations are done to confirm the diagnosis prior to labeling as textiloma as it is a critical diagnosis with medicolegal importance which can lead to mental agony, humiliation, huge financial compensation and imprisonment on surgeon's part while increased morbidity, mortality and financial loss on patient's part as a sequela of a medicolegal battle between a patient and a negligent surgeon. Pathologically it is mostly diagnosed incidentally when fine-needle aspiration cytology or biopsy is done when a patient presents as a mass [12-14]. Histopathological findings in such cases can present as inflammatory response cells with or without granuloma formation and/or degenerating fabric threads/hemostatic particles. Internal areas of necrosis can also be seen on histopathology $[13,14]$. Imaging modalities are the most reliable and frequently used mode of investigations for the diagnosis of musilinomas.

Ultrasound findings may range between a heterogeneous solid mass or a cystic lesion with an internal heterogeneous component [15]. It may also present as an echogenic area with dense posterior acoustic shadowing which remains unchanged upon varying the ultrasound beam parameters [15]. Most abdominal sponges used in operation theatres have a metallic marker in them therefore, an $\mathrm{X}$-ray of the surgical site may be the only diagnostic investigation required for confirmation of diagnosis [16]. Seldomly in the case of radiolucent sponges, may present as a low-density mass with illdefined internal air bubbles which are distinct from the normal gas in that region [17]. Gossypiboma is frequently seen on CT during investigations carried out for masses or abscesses thus on CT it appears predominantly as a heterogeneous mass with internal whorl like pattern which is encapsulated or may have a calcified rim whereas peripheral enhancement of the mass is seen when contrast is administered $[18,19]$. Fluid collection or air bubbles may be present depending upon the elapsed time since surgery $[18,19]$.
Fluoroscopy is also a diagnostic modality for detecting intraluminal textilomas or those causing extrinsic compression of the intestinal lumen thus causing an intestinal obstruction [20,21]. Other investigations that may reveal gossypiboma include MRI, where it appears as a mass with a hyper intense center and a hypo intense rim showing avid contrast enhancement [22]. Endoscopy, colonoscopy and sigmoidoscopy can also be helpful in the diagnosis of intraluminal gossypiboma [23]. Complications of retained gauze piece include abscess formation, intestinal obstruction, malabsorption, hemorrhage, fistula formation, pseudotumor formation causing mass effects, etc. Management of such cases is always surgical ranging from simple removal of the retained foreign body till wide surgical dissection with gut resection in complicated cases.In some cases endoscopic removal is also possible and retained gauze can be removed endoscopically [23]. In conclusion, as it is wisdom from the old proverbs that prevention is better than cure still holds good in this case. When one can prevent it has to be emphasized that such incidents should be prevented by any means because of its psychosocial effects on doctors as well as patients. In this instance a few recommendations that are to be considered including wise planning of the workload, adequate and trained staff with specific roles.

The theatre environment should be conducive to concentration with minimal in and out traffic and an obligation to carry a single surgery in a single theatre room. Surgical sponge count at four intervals should be mandatory before and after the surgery and has to be documented on notice boards by the paramedical team leader and verified by the surgeon in the operation theatre along with patient data so as to recheck at the end of surgery. For every emergency sponge addition, the number should be documented separately. Another important low cost, quick and effective technique on the table is to use a pack of three to five abdominal sponges instead of solitary sponge or gauze whenever a cavity is opened and the ends of those sponges should be tied to each other with a knot as it is physically unlikely to leave a pack of five sponges 
in a cavity [24]. Once saturated they can be removed and replaced by another similar pack [24]. Moreover, single gauze pieces with no radiopaque marker should be dipped in iodinated contrast and post-operatively at least a detailed ultrasound examination followed by an X-ray or CT scan if needed should be carried out prior to discharge so that the early detection could be done instead of detecting the cases after decades. Once it occurs proper documentation should be done and evaluation must be carried out to rectify the negligence level as it is not always the surgeon who is responsible but consequences are merely faced by the surgeon alone. Few modern techniques include the article Electronic Surveillance System (EAS) and Radiofrequency identification microchips which are costly yet the chances of error are minimal as compared to human counting errors $[25,26]$.

\section{References}

1. Kim HS, Chung TS, Suh SH, Kim SY (2007)MR imaging findings of paravertebral gossypiboma. AJNR Am J Neuroradiol 28(4): 709-713.

2. Kiernan F, Joyce M, Byrnes CK, O Grady H, Keane FB (2008) Gossypiboma: a case report and review of the literature. Ir J Med Sci. 177(4): 389-391.

3. Rajput A, Loud PA, Gibbs JF, Kraybill WG (2003) Diagnostic challenges in patients with tumors: case 1. Gossypiboma (foreign body) manifesting 30 years after laparotomy. Journal of Clinical Oncology 21(19): 37003701.

4. Dakubo J, Clegg Lamptey J, Hodasi W, Obaka H, Toboh H (2009) An intraabdominal gossypiboma. Ghana medical journal 43(1): 43-45.

5. Sun HS, Chen SL, Kuo CC, Wang SC, Kao YL (2007) Gossypiboma-retained surgical sponge. Journal of the Chinese Medical Association70(11): 511513.

6. Cruz RJ Jr, Poli de Figueire do LF, Guerra L (2003) Intracolonic obstruction induced by a retained surgical sponge after trauma laparotomy. J Trauma 55(5): 989-991.

7. Moslemi MK, Abedinzadeh M (2010) Retained Intraabdominal Gossypiboma, five years after bilateral orchiopexy. Case Report Med 2010: 420357

8. Lv YX, Yu CC, Tung CF, Wu CC (2014) Intractable duodenal ulcer caused by transmural migration of gossypiboma into the duodenum--a case report and literature review. BMC Surg 14: 36

9. Kabiri H, Caidi M, Benamor J, El Mazlout A, Benosman A (2001) Intrathoracic textiloma caused by transdiaphragmatic migration after biliary surgery: report of a case. Rev Pneumol Clin 57(5): 362-365.

ISSN: $2574-1241$

DOI: $10.26717 /$ BJSTR.2020.26.004417

Christopher Antwi. Biomed J Sci \& Tech Res

This work is licensed under Creative

Commons Attribution 4.0 License

Submission Link: https://biomedres.us/submit-manuscript.php
10. Biswas, Rabi Sankar (2012) Gossypiboma and surgeon- current medicolegal aspect - a review. The Indian journal of surgery 74(4): 318322.

11. Wan Y, Ko S, Ng K (2004) Role of CT-guided core needle biopsy in the diagnosis of a gossypiboma: case report. Abdom Imaging 29(6): 713715 .

12. Kato M, Kawahara Y, Matsuda K, Tajiri H(2015) Gossypiboma diagnosed by a forward-view endoscopic ultrasound fine-needle aspiration out of the sigmoid colon. Endosc Ultrasound 4(2): 149-151.

13. Choi B, Kim SH, Yu ES, Chuang HS, Han MC (1988) Retained surgical sponge: Diagnosis with CT and Sonography. AJR 150(5): 1047-1050.

14. Angus RO Connor, Fergus V Coakley, Maxwell V Meng, Stephen Eberhardt (2003) Imaging of Retained Surgical Sponges in the Abdomen and Pelvis. American Journal of Roentgenology 180(2): 481-489.

15. Shyung LR, Chang WH, Lin SC, Shih SC, Kao CR (2005) Report of gossypiboma from the standpoint in medicine and law. World J Gastroenterol11(8): 1248-1249.

16. Malik A, Jagmohan P. Gossypiboma: US and CT appearance. Indian J Radiol Imaging 12(4):503-504

17. ST Young, EK Paulson, RL Mc Cann, ME Baker (1993) Appearance of oxidized cellulose (Surgicel) on postoperative CT scans: similarity to postoperative abscess. American Journal of Roentgenology 160(2): 275277.

18. Sharma D, Pratap A, Tandon A, Shukla RC, Shukla VK (2008) Unconsidered cause of bowel obstruction-gossypiboma. Can J Surg51(2): E34-E35.

19. Ogundiran T, Ayandipo O, Adeniji Sofoluwe A, Ogun G, Oyewole O (2011) Gossypiboma: complete transmural migration of retained surgical sponge causing small bowel obstruction. BMJ Case Rep. 2011:bcr0420114073.

20. Chavan R, Lakhtakia S, Nabi Z, Gupta R, Reddy DN (2018) Endoscopic management of gossypiboma. VideoGIE3(10): 306-307.

21. Srivastava A, Kataria K, Chella VR (2014) Prevention of gossypiboma. Indian J Surg 76(2): 169.

22. A Macario, D Morris, S Morris (2006) Initial clinical evaluation of a handheld device for detecting retained surgical gauze sponges using radiofrequency identification technology. Archives of Surgery 141(7): 659-662.

23. CE Fabian (2005) Electronic tagging of surgical sponges to prevent their accidental retention. Surgery, 137(3): 298-301.

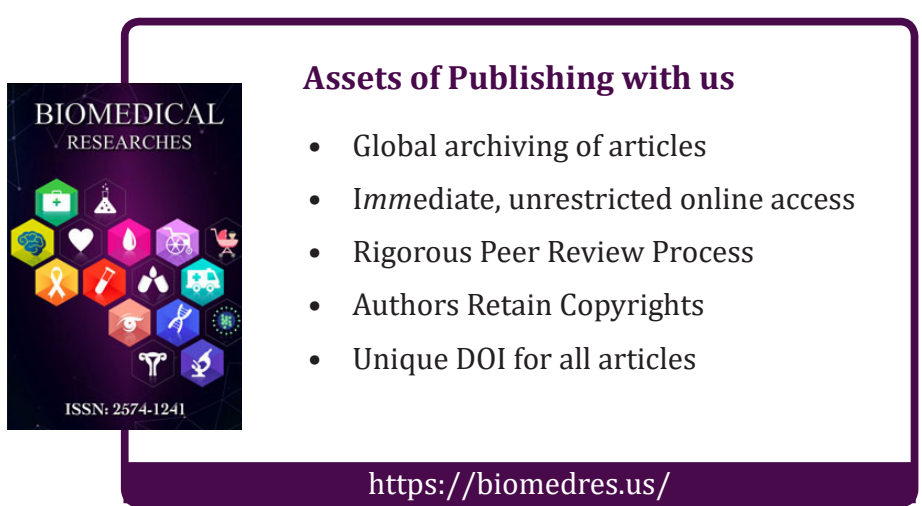

